\title{
Stress-Strain response of irradiated material using dislocation dynamics modelling
}

\section{B.K. Dutta}

Reactor Safety Division, Bhabha Atomic Research Centre

Trombay, Mumbai, India-400 085.

E-mail: bkdutta@barc.ernet.in

\section{P.V. Durgaprasad}

Reactor Safety Division, Bhabha Atomic Research Centre

Trombay, Mumbai, India-400085.

E-mail: pvdp@barc.ernet.in

Irradiation of materials by energetic particles causes significant degradation of the mechanical properties, most notably an increased yield stress and decreased ductility, thus limiting lifetime of materials used in nuclear reactors. Effect of irradiation on materials is to produce vacancies and self-interstitial atoms, and stacking fault tetrahedra. These defect clusters form loops around existing dislocations, leading to their decoration and immobilization. These microstructural features ultimately lead to radiation hardening in most of the materials. An attempt is made to understand these phenomena using discrete dislocation dynamics (DD) modelling. The plastic flow is represented by collective motion of a large number of edge dislocations. The dislocation fields are specified by continuum elasticity theory. Since the elastic fields act infinite in medium, corrections for boundaries are specified by a complementary problem, which consists of solving a linear elastic boundary value problem through finite element method. The dislocation phenomenon like annihilation, generation and pinning of dislocations by obstacles are incorporated in the model. Irradiation effects are modelled by assuming that a fraction of total dislocations are locked by irradiation induced defects. These dislocations get unlocked when stress on them exceeds a threshold value. The stress-strain response of an irradiated material as a function of density of Frank-Read sources and obstacles density and irradiation fluence is studied.

International Conference on Statistical Mechanics of Plasticity and Related Instabilities Indian Institute of Science, Bangalore August 29-September 2, 2005

\footnotetext{
* B.K. Dutta. Tel.: +91-022-25593778.
} 


\section{Introduction}

The role of dislocations in material plastic deformation behavior is well established. In unirradiated materials, the dislocation-dislocation interactions determine the characteristics of plastic deformation, where the dislocation motion is impeded by the sessile junctions or dipoles. However, for irradiated materials, interaction of dislocations with irradiation-induced defects entirely controls the plastic yield. The microstructure of irradiated materials evolves over a wide range of length and time scales, making radiation damage and inherently multi-scale phenomenon. At the atomic length scale, cascade induced atomic displacements give rise to a highly non-equilibrium concentration of defects like vacancies, interstitials and stacking fault tetrahedra (SFT). Over macroscopic length scale, these defects can alter the microstructure causing significant degradation of mechanical and other properties. The main notable features of irradiation-induced mechanical behavior are: an increased yield strength with irradiation dose, and an instability that results in plastic flow localization within dislocation channels leading to loss of ductility and premature failure.

Here, an attempt is made to understand these phenomenons using the dislocation dynamics modelling. An in-house code for two-dimensional discrete dislocation dynamics (DD) analysis is developed based on formulation given in [1]. Here, stresses and strains are written as superposition of fields due to the discrete dislocations and complementary fields that enforce the boundary conditions. This leads to a linear elastic complementary boundary value problem, which is solved by finite element method. This code is used to study small strain plasticity problems. The organization of the paper is as follows: In section 2, brief formulation for discrete dislocation analysis is presented. In section 3, stress-strain response of unirradiated Aluminium is discussed. Predicted stress-strain curves for irradiated copper are presented in section 4.

\section{Formulation for discrete dislocation analysis}

The problem is formulated as follows: Consider a linear elastic body of volume $\mathrm{V}$ which contains a distribution of dislocations. The dislocations are treated as line defects in the elastic continuum. Each dislocation is characterized by its Burger's vector $b^{i}$ and its slip plane. The shear modulus of the material is $\mu$. The body is subjected to time dependent traction and displacement boundary conditions $\mathrm{T}=\mathrm{T}_{\mathrm{o}}(\mathrm{t})$ on $\mathrm{S}_{\mathrm{f}}$ and $\mathrm{u}=\mathrm{u}_{\mathrm{o}}(\mathrm{t})$ on $\mathrm{S}_{\mathrm{u}}$. The deformation process will lead to the motion of dislocations, mutual annihilation and generation of new dislocations and their pinning at point obstacles. The obstacles may be second phase particles, defects generated due to irradiation etc. The analysis of deformation process is performed in an incremental manner in time, where the incremental step at any instant $t$ involves three main computational stages: (i) determining the current stress and strain state of for the current dislocation arrangement; (ii) determination of the so-called Peach-Koehler force, i.e., the driving force for changes in dislocation structure; and (iii) determination of the instantaneous rate of change of dislocation structure on the basis of a set of constitutive equations of motion, annihilation and generation of dislocations. All the three stages of computatation are described below. 
The current state of body in terms of the displacement, strain and stress fields is written as the superposition of two fields,

$$
u=\tilde{u}+\hat{u} \quad \varepsilon=\tilde{\varepsilon}+\hat{\varepsilon} \quad \sigma=\tilde{\sigma}+\hat{\sigma} \text { in } V
$$

respectively, as illustrated in Fig. 1. The ( $\left(^{(}\right)$fields are associated with the $n$ dislocations in the current configuration but in infinitely large medium of material. These fields are obtained by superposition of the fields associated with each individual dislocation,

$$
\tilde{u}=\sum_{i=1}^{n} u^{i} \quad \tilde{\varepsilon}=\sum_{i=1}^{n} \varepsilon^{i} \quad \tilde{\sigma}=\sum_{i=1}^{n} \sigma^{i}
$$

The corresponding stress $(\tilde{\sigma})$ and displacement $(\tilde{u})$ fields of a dislocation [1] are given by:

$$
\begin{aligned}
& u_{1}^{i}=\frac{b^{i}}{2 \pi(1-v)}\left\{\frac{1}{4} \frac{\pi \Delta \xi_{2} \sin \pi \Delta \xi_{1}}{\cosh \pi \Delta \xi_{2}-\cos \pi \Delta \xi_{1}}-(1-v) \tan ^{-1}\left(\frac{\tan \pi \Delta \xi_{1} / 2}{\tanh \pi \Delta \xi_{2} / 2}\right)\right\} \\
& -\frac{b^{i}}{2} \delta^{i} \operatorname{sign}\left(\Delta \xi_{2}\right) \\
& u_{2}^{i}=\frac{b^{i}}{2 \pi(1-v)}\left\{\frac{1}{4} \frac{\pi \Delta \xi_{2} \sinh \pi \Delta \xi_{2}}{\cosh \pi \Delta \xi_{2}-\cos \pi \Delta \xi_{1}}\right. \\
& \left.-\frac{1}{4}(1-2 v) \ln \left(\cosh \pi \Delta \xi_{2}-\cos \pi \Delta \xi_{1}\right)\right\} \\
& \sigma_{11}^{i}\left(x_{\alpha}\right)=-\frac{\mu}{2 \pi(1-v)} \frac{\pi b^{i}}{2 w} \frac{1}{\cosh \pi \Delta \xi_{2}-\cos \pi \Delta \xi_{1}} \\
& \times\left\{2 \sinh \pi \Delta \xi_{2}+\pi \Delta \xi_{2} \frac{1-\cos \pi \Delta \xi_{1} \cosh \pi \Delta \xi_{2}}{\cosh \pi \Delta \xi_{2}-\cos \pi \Delta \xi_{1}}\right\} \\
& \sigma_{22}^{i}\left(x_{\alpha}\right)=\frac{\mu}{2 \pi(1-v)} \frac{\pi b^{i}}{2 w}\left\{\pi \Delta \xi_{2} \frac{1-\cos \pi \Delta \xi_{1} \cosh \pi \Delta \xi_{2}}{\cosh \pi \Delta \xi_{2}-\cos \pi \Delta \xi_{1}}\right\} \\
& \sigma_{12}^{i}\left(x_{\alpha}\right)=\frac{\mu}{2 \pi(1-v)} \frac{\pi b^{i}}{2 w} \frac{\sin \pi \Delta \xi_{1}}{\cosh \pi \Delta \xi_{2}-\cos \pi \Delta \xi_{1}} \\
& \times\left\{1-\pi \Delta \xi_{2} \frac{\sinh \pi \Delta \xi_{2}}{\cosh \pi \Delta \xi_{2}-\cos \pi \Delta \xi_{1}}\right\}
\end{aligned}
$$

where, $\Delta \xi_{\alpha}=\left(x_{\alpha}-X_{\alpha}^{i}\right) / w \quad(\alpha=1,2), u_{\alpha}^{i}(\alpha=1,2)$ are the displacements due to dislocation $i$ at $(\mathrm{x}, \mathrm{y})$. The term with $\delta^{i}$ in eqn. (3) ensures that the solution is valid for $\Delta \xi_{1} \in(-1,1)$, by letting

$$
\delta^{i}=\left\{\begin{array}{lc}
-1 & \text { if } \Delta \xi_{1} \in(-3 / 2,-1 / 2) \\
+1 & \text { if } \Delta \xi_{1} \in(1 / 2,3 / 2)
\end{array}\right.
$$

Because of periodic boundary conditions, dislocation leaving the cell at $x_{1}= \pm w$ will re-enter at the opposite side $x_{1}=\mp w$. Only the displacement fields of the dislocation need to be corrected for that by adding the contribution 


$$
u_{1}^{i}\left(x_{\alpha}\right)=\frac{b^{i}}{2} \delta^{i} \operatorname{sign}\left(\Delta \xi_{2}\right) .
$$

As the solution for ( ) fields is facilitated by virtue of absence of boundaries, the $\left(^{\wedge}\right)$ fields are added to correct for the actual boundary conditions on S. This leads to a linear elastic complementary boundary value problem, the governing equations of which are given by,

$$
\left.\begin{array}{c}
\left.\begin{array}{c}
\nabla . \hat{\sigma}=0 \\
\hat{\varepsilon}=\nabla \hat{u}
\end{array}\right\} \text { in } V \\
\hat{T}=T_{o}-\tilde{T} \text { on } S_{f} \\
\hat{u}=u_{o}-\tilde{u} \text { on } S_{u}
\end{array}\right\} b . c^{\prime} s
$$

Solution to this complementary boundary value problem is obtained using finite element method. The motion of the dislocations is governed by constitutive equations according to liner drag relation given by,

$$
\tau^{i} b^{i}=B v^{i}
$$

where, $\tau^{i} b^{i}=f^{i}$ is the Peach-Koeheler force, B is the drag coefficient and $v$ is the velocity of a dislocation. The result of the above formulation is a set of non-linear first order differential equations governing the motion of the dislocations, which are solved using Euler forward time integration method.

The motion of dislocations along a slip plane can be hindered in real crystals by obstacles such as dislocations on intersecting slip planes, small precipitates etc. We model this by means of point obstacles at which moving dislocations get pinned down. Such pinned dislocations will be released when the resolved shear strength on them exceeds the obstacle's strength $\left(\tau_{\mathrm{obs}}\right)$. Two edge dislocations with opposite Burger's vector will annihilate each other when they are brought closer together within a critical annihilation distance $L_{e}$. New dislocations are being generated through the operation of Frank-Read sources. We assume that sources are point sources on the slip plane, which generate a dislocation dipole when the magnitude of the shear stress exceeds the critical stress $\left(\tau_{\text {nuc }}\right.$ ) during a period of time $t_{\text {nuc }}$. The distance $L_{n u c}$ (see [1]) between the two dislocations is determined by the critical stress according to

$$
L_{\text {nuc }}=\frac{\mu}{2 \pi(1-v)} \frac{b}{\tau_{\text {nuс }}} .
$$

\section{Stress-strain response for unirradiated Aluminium under simple shear}

The DD model described above is used to study deformation behavior of different grades of Aluminium under simple shear. For this case, no irradiation effects are assumed. Different grades of Aluminium are characterized by density of obstacles, density of nucleation sources. The simulation cell of $\mathrm{Al}$ is assumed to be of dimensions $2 w \times 2 h$, where $w=h=1 \mu \mathrm{m}$. The shear modulus $\mu=26 \mathrm{Gpa}$ and the Poisson' ratio is $v=0.3$. The drag coefficient $B$ in eqn.(10) is taken as $10^{-4}$ Pa.s. The plastic flow is represented by a collection of large number of edge dislocations.We assume that the material consists of randomly distributed defect structure, such as point obstacles, Frank-read dislocation nucleation sources. The material is assumed to have a 
initial dislocation density of $\rho_{\text {disinit }}=200 / \mathrm{hw}$, which is then relaxed. During relaxation, the dislocations will interact with each other and try to attain equilibrium position. In the relaxed configuration, obstacles and nucleation sources are randomly generated. All the obstacles are assumed to have same strength $\tau_{\mathrm{obs}}=5.7 \times 10^{-3} \mu$. The strength of sources is selected randomly from a Gaussian distribution with a mean strength of $\bar{\tau}_{n u c}=1.9 \times 10^{-3} \mu$ corresponding to a mean nucleation distance of $L_{n u c}=125 \mathrm{~b}$ and the nucleatin time is taken as $t_{n u c}=2.6 \times 10^{6} \mathrm{~B} / \mu$. The strength distribution is assumed to have a standard deviation of $0.2 \bar{\tau}_{\text {nuc }}$. The critical annihilation length is taken as $L_{e}=6 b$. The unit cell is then subjected to simple shear along top and bottom edges in time incremental manner, with a strain rate of $\dot{\Gamma}$,

$$
\left.\begin{array}{l}
u_{1}(t)= \pm h \dot{\Gamma} t \\
u_{2}(t)=0
\end{array}\right\} \quad \text { along } x_{2}= \pm h
$$
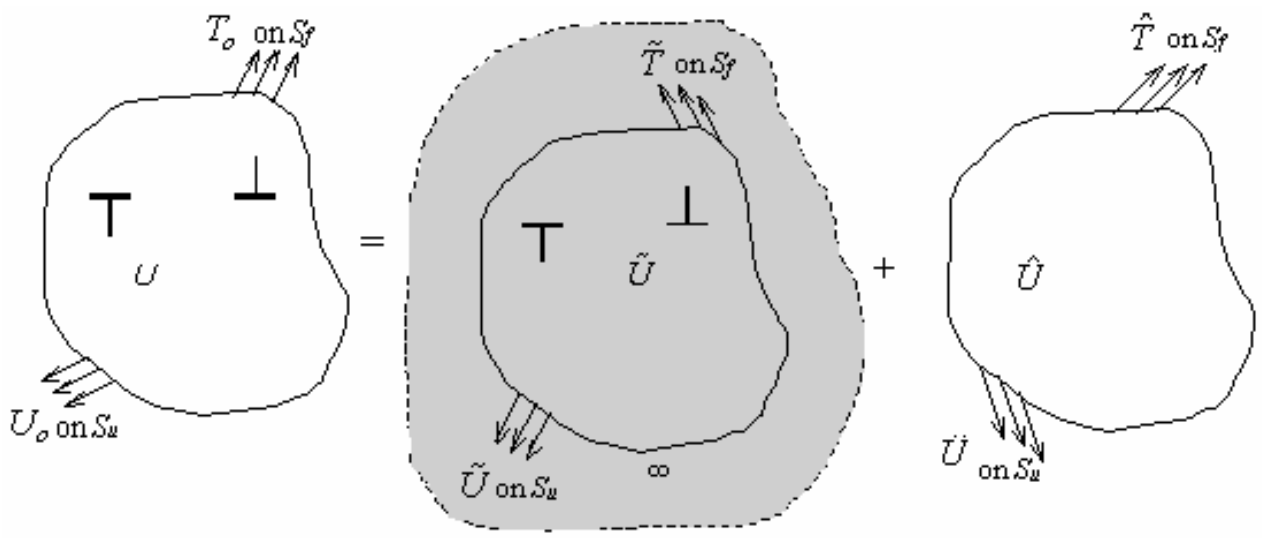

Fig. 1 Decomposition of problem into problem of dislocations in infinite solid ( fields) and complementary problem without dislocation fields (^ fields)

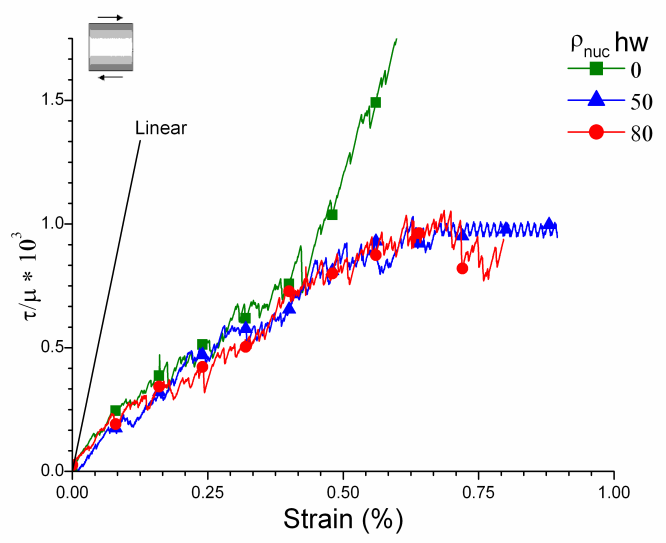

(a)

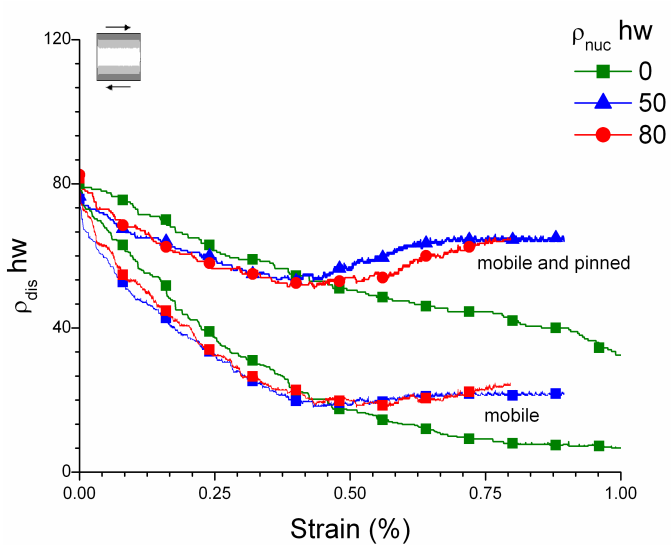

(b)

Fig. 2 Effect of density of nucleation sources on (a) shear response (b) evolution of total and mobile dislocation density 


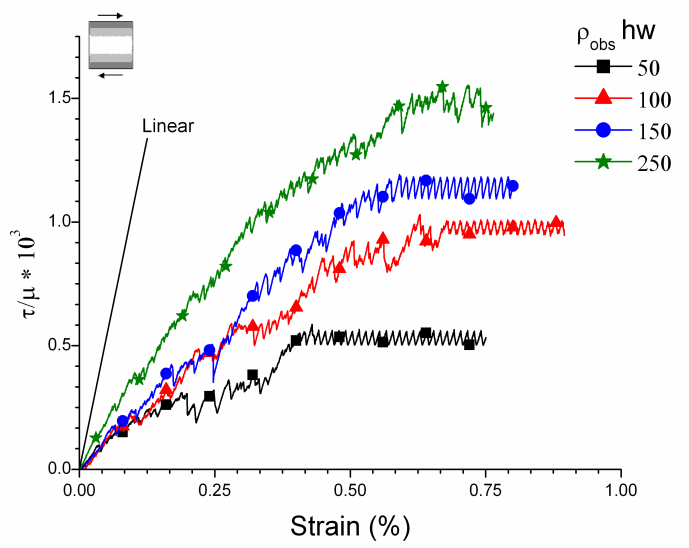

(a)

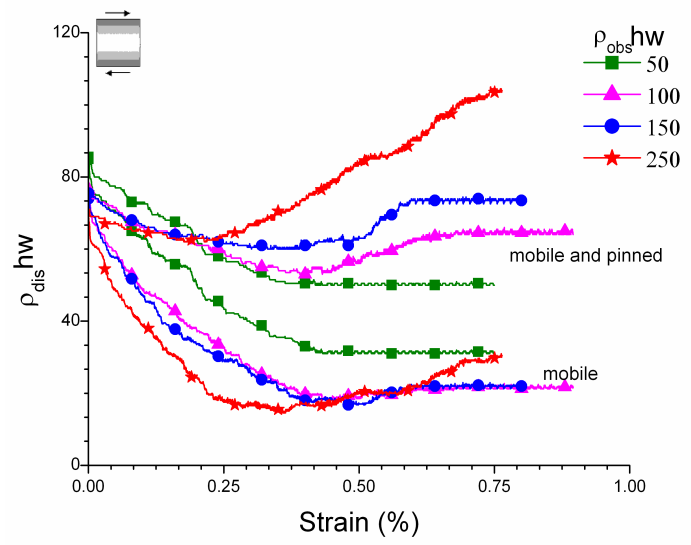

(b)

Fig. 3 Effect of obstacle density on (a) shear response (b) evolution of total and mobile dislocation density

Fig. 2(a) shows effect of source density $\rho_{\text {nuc }}$ on shear response of Aluminum. A constant obstacle density of $\rho_{\mathrm{obs}}=100 / \mathrm{hw}$ is assumed for this case. When source density is zero, shear deformation is mainly controlled by annihilation process and response tends to become purely elastic. With sufficient sources, dislocation generation overcomes annihilation process at a strain of about $0.5 \%$. The rate of dislocation generation then appears to be independent of source density and will be controlled by strength of the sources. Fig. 2(b) shows the evolution of dislocation densities during deformation process.

Fig. 3(a) shows the effect of obstacle density on shear response of Al. The source density is fixed as $\rho_{\text {nuc }}=50 / \mathrm{hw}$. It clearly shows an increase in strain hardening due to an increase in obstacle density. With highest obstacle density $\left(\rho_{\text {obs }}=250 / \mathrm{hw}\right)$, there is a strong initial strain hardening as compared to other three cases with lower obstacle densities. Fig. 3(b) shows effect of obstacle density on evolution of dislocation density. The case with lowest obstacle density shows monotonic decrease of dislocation density (total and mobile) until steady state value is reached. Correspondingly, stress-strain response shows a gradual transition to flow. As obstacle density increases, dislocation densities increase after some deformation indicating the decrease of annihilation effects. With largest obstacle density, annihilation becomes highly inactive and there is a steep increase in dislocation density, consequently, there is a strong strain hardening. After some deformation, stress-strain response reaches a steady state value and dislocations found stable values.

\section{Stress-strain response for irradiated Copper under simple shear}

Here, we consider the problem of irradiation-induced hardening in Copper. Plastic deformation and hardening in irradiated materials is controlled primarily by the defects due to irradiation (vacancies, self-interstitial atoms and SFT's) and their interaction with dislocations. These defect clusters will tend to form loops around existing dislocations, leading to their decoration and immobilization. In order to understand the effect of this phenomenon on 
deformation behavior, we consider irradiated copper under simple shear using two-dimensional DD model described in section 2. In spite of the fact that this phenomenon is fully three dimensional in nature, the two-dimensional model can be used to understand basic mechanism of irradiation hardening.

The simulated copper cell is assumed to be of dimensions $2 \mu m \times 2 \mu m$. The shear modulus is $\mu=55 \mathrm{GPa}$. The plastic flow is represented by a collection of large number of edge dislocations. We assume that the material consists of randomly distributed defect structure such as point obstacles, Frank-Read dislocation nucleation sources. The irradiation induced hardening may be understood in terms of cascade induced source hardening in which the dislocations are considered to be locked by the loops decorating them[2,3]. The density and strength of these defect loops depend on the fluence level. To mimic these irradiation effects, we assume that some fraction of total dislocations (characterizing the fluence) are locked by the defect loops. When the stress on the dislocations exceeds a critical value, they become free to move on their glide planes. For our analysis, we have taken a critical stress value of $50 \mathrm{MPa}$ for all the defect loops. The motion of the dislocations is governed by eqn.(10). During gliding, any dislocation may get pinned down by the point obstacles or may get annihilated by an opposite dislocation. New dislocations will be generated by Frank-Read mechanism, which is mimicked here by point sources. These sources will nucleate a dislocation dipole when stress on a source exceeds a critical value.

Initially, the material is assumed to have a dislocation density of $\rho_{\text {disinit }}=200 / \mathrm{hw}$, which is then relaxed. During relaxation, the dislocations will interact with each other and try to attain equilibrium position. In the relaxed configuration, obstacles and nucleation sources are randomly generated. Two different problems with densities (a) $\rho_{\text {nuc }}=50 / \mathrm{hw}, \rho_{\mathrm{obs}}=100 / \mathrm{hw}$, (b) $\rho_{\text {nuc }}=100 / \mathrm{hw}, \rho_{\text {obs }}=200 / \mathrm{hw}$ are analyzed. The number of dislocations that are decorated by irradiation-induced defects is given by $\rho_{\text {irrd }}$. The effect of irradiation fluence is characterized here by $\rho_{\mathrm{f}}=\rho_{\text {irrd }} / \rho_{\text {dis }}$-the fraction of total dislocations being decorated or locked by the irradiation-induced defects. This forms the initial dislocation configuration for further analysis. The material is then subjected to simple shear conditions in time incremental manner at top and bottom edges and stress-strain response is obtained.

Typical predicted stress-strain curves for both the problems are shown in Fig.4 revealing the effect of dislocation loops in increased yield stress and increased hardening. For problem (a), when $\rho_{\mathrm{f}}$ is increased from 0.0 to 0.6 , yield stress (shear) increases from $8 \mathrm{MPa}$ to $14 \mathrm{MPa}$. Problem (b) also exhibits similar trend, but numerical values do not differ much from problem (a). This may be due to increase in the density of nucleation sources, which generate more mobile dislocations compensating for more point and irradiation obstacles. The corresponding dislocation configurations at i) zero strain and ii) after shearing upto $0.8 \%$ strain for the problem (b) are shown in Fig. 5. Even though current model is two-dimensional and considers only edge dislocations, the results illustrate the potential of DD simulations in providing answers to a number of critical questions related irradiation induced hardening and associated phenomena like plastic flow localization etc. Detailed analysis of irradiation effects using three-dimensional DD modelling with curved dislocations is under progress. 


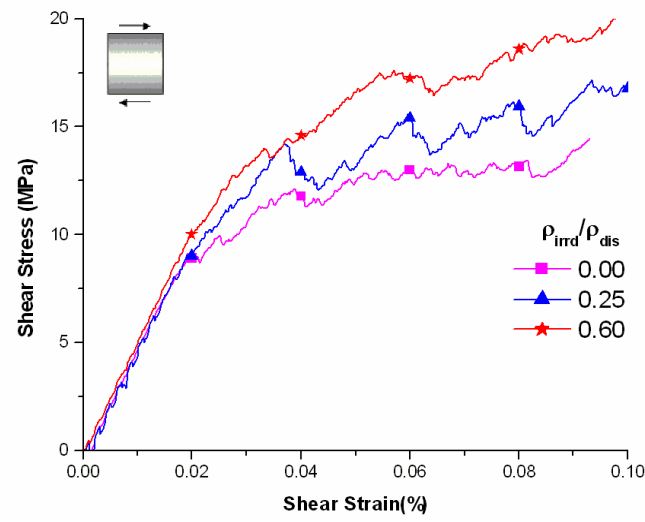

(a) $\mathrm{nsrc}=50 / \mathrm{hw} ;$ nobs $=100 / \mathrm{hw}$

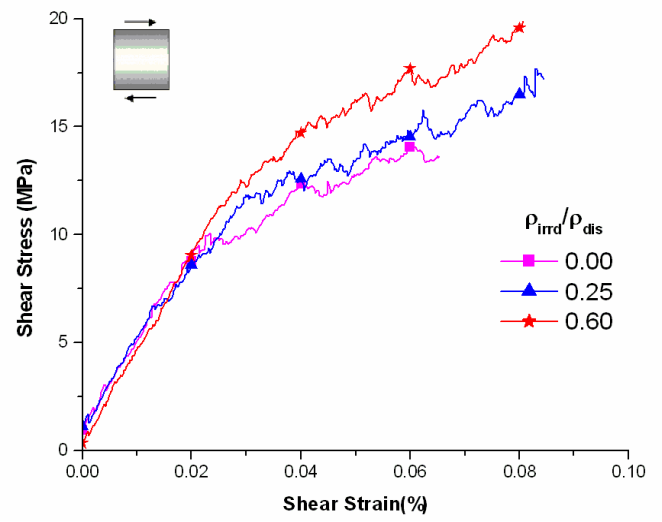

(b) $\mathrm{nsrc}=100 / \mathrm{hw}$; nobs $=200 / \mathrm{hw}$

Fig. $4(\mathbf{a \& b})$ Stress-strain plot showing irradiation effects. Fluence level is characterized by the ratio $\rho_{\mathrm{f}}=\rho_{\text {irrd }} / \rho_{\mathrm{dis}}$ which gives fraction of total dislocations being decorated by irradiation-induced defects.

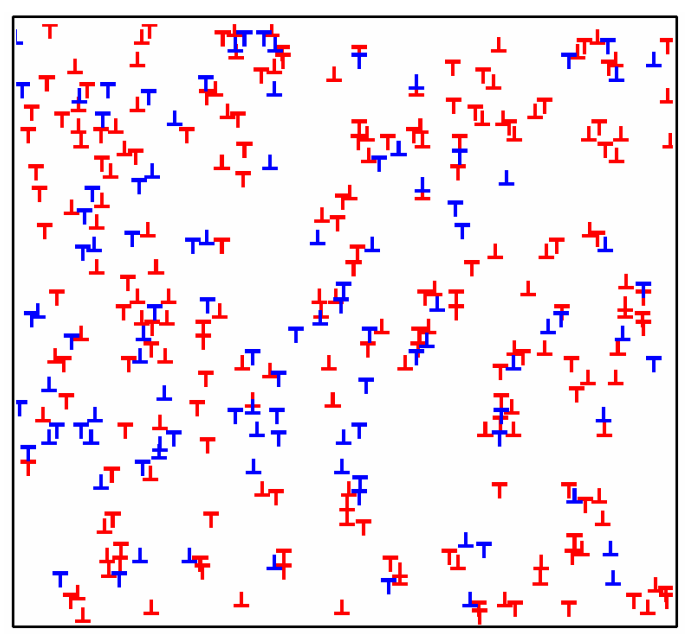

(a) initial

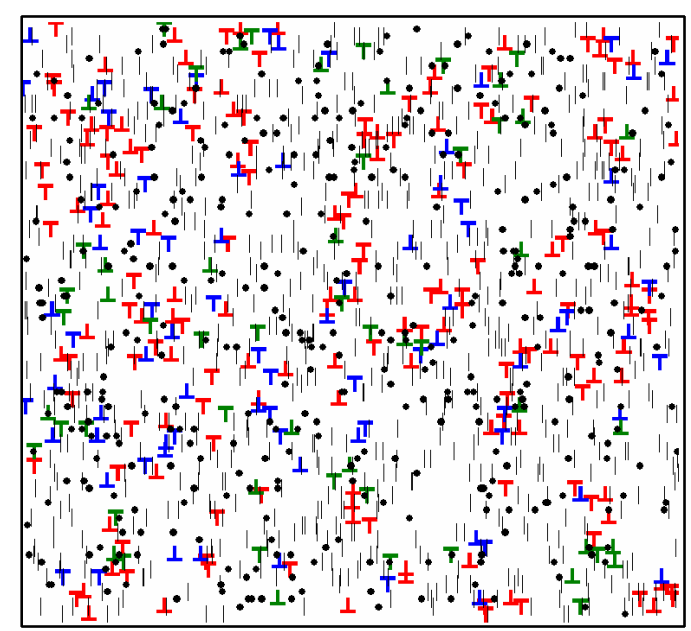

(b) at strain $0.8 \%$

Fig. 5 a) Initial dislocation configuration shown. $n s r c=100 / \mathrm{hw}$ and nobs $=200 / \mathrm{hw}$. The dislocations which are being decorated by irradiation defects are shown red in color $(\perp T)$ and remaining are shown blue in color $(\perp \mathbf{T})$. b) dislocation configuration at a strain of $0.8 \%$. New dislocations that are generated during evolution are shown green in color $\left(\begin{array}{l}\perp \\ \mathbf{T}\end{array}\right)$. Frank-Read sources $(\bullet)$ and obstacles $(\mid)$ are also shown.

\section{Conclusion}

The irradiation induced hardening in materials is studied using the in-house twodimensional discrete dislocation dynamics code. Under irradiation, dislocation loops attract mobile defect clusters and gets pinned down. Such dislocations require a critical stress to get unlocked before they can move on their glide planes under external stress. These phenomenon are modeled by taking some fraction of dislocations being decorated by such irradiation-induced 
defects thus characterizing the irradiation fluence. The other dislocation phenomena like annihilation, nucleation, pinning down by obstacles are also incorporated. Effect of irradiation fluence on stress-strain response of copper under simple shear is determined. Parametric study with respect to density of obstacles and dislocation nucleation sources has been done.

\section{References}

[1] E.V. Giessen, A.Needleman, Modelling and Simul. Mater. Sci. Eng. 3 (1995) 689-735.

[2] H. Trinkaus, B.N. Singh, A.J.E. Foreman, J. Nucl. Mater. 251 (1997) 172.

[3] H.M. Zbib, T.D. Rubia, M. Rhee, J.P. Hirth, J. Nucl. Mater. 276 (2000) 154-165. 